\title{
Investigation of Boron Delta-Layers in Silicon Measured by Atom Probe Tomography (APT)
}

\author{
C. Klein *, S. Mutas **, A. Würfel *** and E. Zschech*
}

* AMD Fab 36 LLC \& Co. KG, Center for Complex Analysis, Wilschdorfer Landstraße 101, 01099 Dresden, Germany

** Fraunhofer-Center Nanoelectronic Technologies, Koenigsbruecker Str. 180, 01099 Dresden, Germany

*** GWT-TUD GmbH, Chemnitzer Straße 48b, 01187 Dresden, Germany

Atom Probe Tomography (APT) is used for three dimensional composition analysis of volumes of typically $100 \times 100 \times 100 \mathrm{~nm}^{3}$ with sub-nm and in many cases even atomic spatial resolution [1]. For the analysis the material has to be formed into the shape of a small tip with a radius of about 50-100 $\mathrm{nm}$. The specimen is then sequentially evaporated atom by atom by applying laser pulses (laser mode) or voltage pulses (voltage mode) to the tip. The reconstruction of Atom Probe data requires knowledge about the tip radius at any time during the measurement. Both in laser pulsing mode and in voltage pulsing mode the radius can be obtained by dividing the tip Voltage $\mathrm{V}$ by a constant factor $\mathrm{k} \times \mathrm{F}$, where $\mathrm{k}$ is a geometrical factor and $\mathrm{F}$ is the evaporation field at the temperature at which the evaporation occurs. It is usually assumed that this factor is constant throughout the measurement. While this is a widely accepted assumption for voltage mode it is less clear under what conditions it holds true in laser mode. Among the materials that are being more and more investigated with laser assisted APT is silicon implanted with boron [2]. Traditionally depth profiles of impurities inside a silicon matrix are obtained with Secondary Ion Mass Spectrometry (SIMS) which is therefore often used to obtain $\mathrm{k} \times \mathrm{F}$ by adjusting the depth scale of APT to the depth scale of SIMS. In order to assess the validity of this method we measured a Si sample that contains Boron doped delta layers at well defined depths. The concentration of Boron was below 1 at $\%$ to make sure that the evolution of the tip shape is governed by the evaporation field of the matrix. Specimens with different shank angles were fabricated with a Focused Ion Beam (FIB) and imaged with a high resolution scanning electron microscope (SEM) before and after the measurement. APT was carried out on a LEAP $3000 \mathrm{X}$ Si microscope at laser pulse energies between $0.1 \mathrm{~nJ}$ and $0.4 \mathrm{~nJ}$. From the reconstruction we obtained values for $\mathrm{k} \times \mathrm{F}$ that best match the known spacing of the boron delta layers as a function of depth and hence for different tip radii. In this way discrepancies between radius measurements obtained from the reconstruction and from SEM imaging can be explained.

References

[1] T.F. Kelly and M. K. Miller, Rev. Sci. Instr. 79 (2007) 031101

[2] P. Ronsheim et al., Appl. Surf. Sci. 255 (2008) 1547 


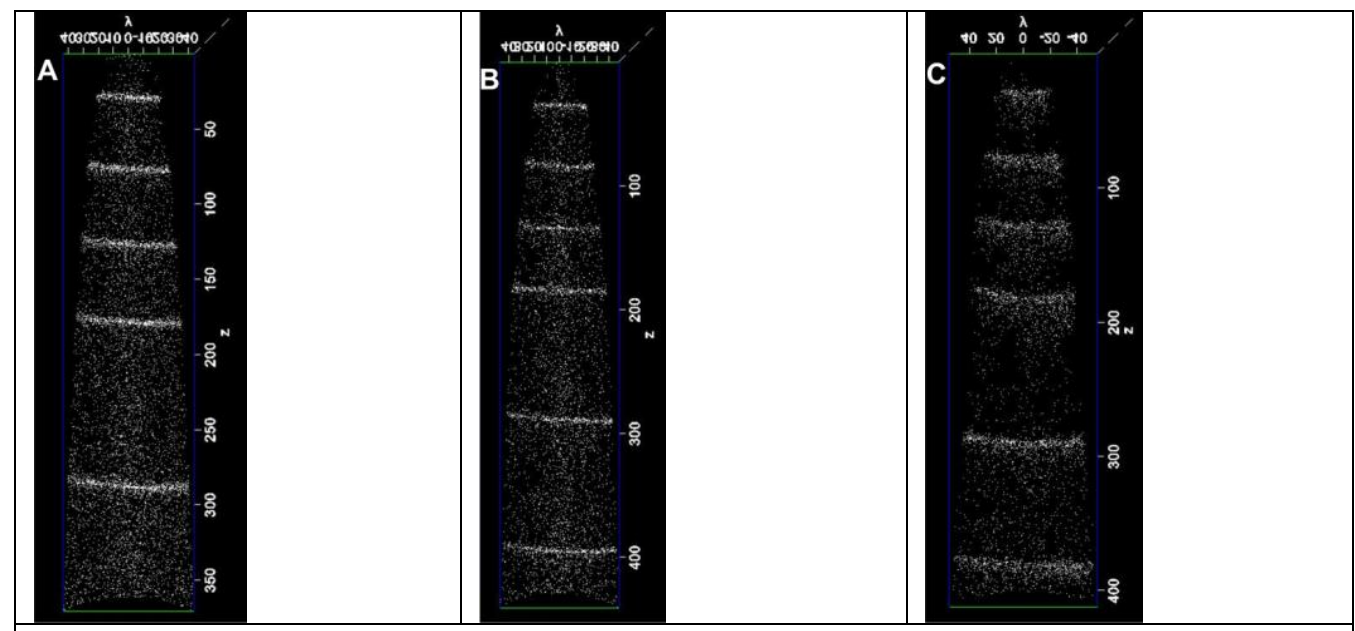

Fig. 1. Reconstruction with adjusted $\mathrm{k} \times \mathrm{F}$ values to obtain correct Boron spacings for laser energies of $0.1 \mathrm{~nJ}(\mathrm{~A}), 0.2 \mathrm{~nJ}(\mathrm{~B})$ and $0.3 \mathrm{~nJ}(\mathrm{C})$.

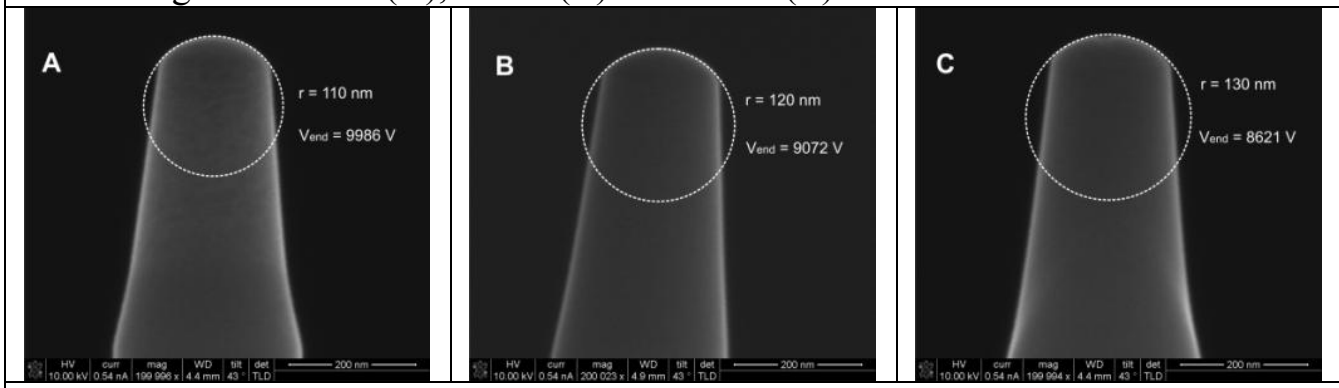

Fig. 2. SEM images after measurements with end radii and end voltages for laser energies of $0.1 \mathrm{~nJ}(\mathrm{~A}), 0.2 \mathrm{~nJ}(\mathrm{~B})$ and $0.4 \mathrm{~nJ}(\mathrm{C})$.

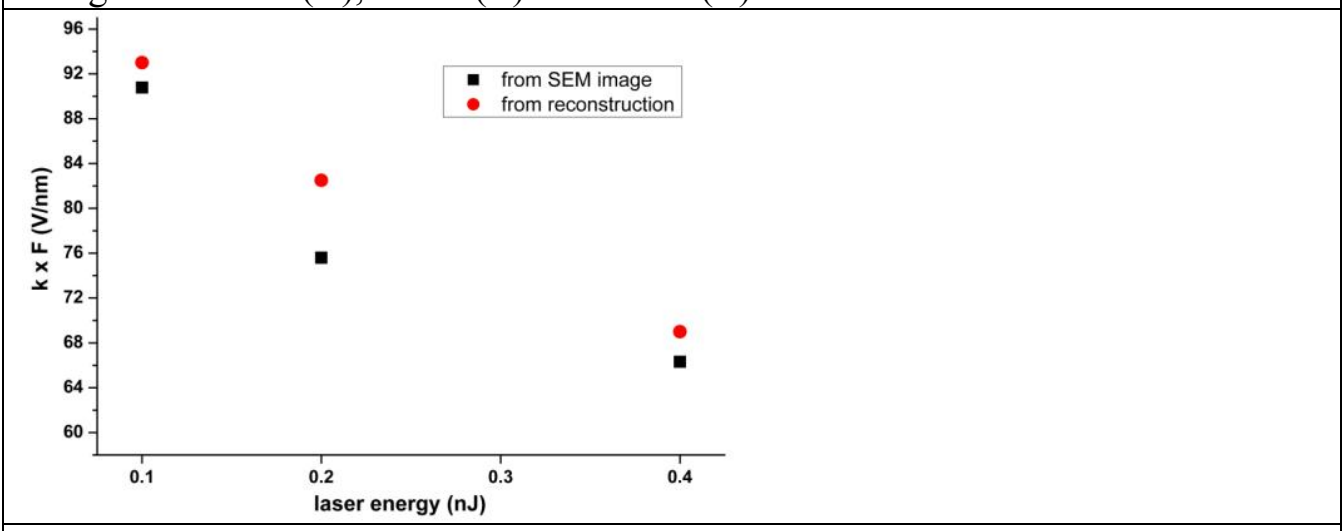

Fig. 3. Comparison of $\mathrm{k} \times \mathrm{F}$ values obtained from SEM images and from reconstruction for correct Boron spacings for different laser energies. 